
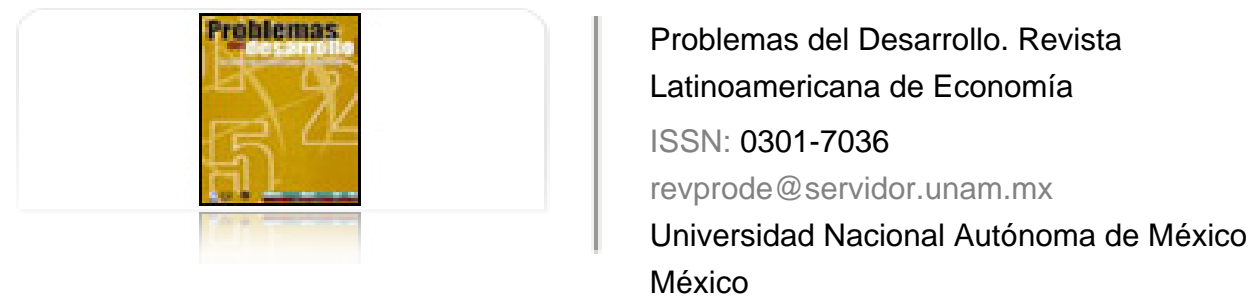

Rodríguez Castellanos, Arturo; Urionabarrenetxea Zabalandikoetxea, Sara; San Martín Albizuri, Nerea Crisis financieras y globalización: un análisis de sus factores determinantes Problemas del Desarrollo. Revista Latinoamericana de Economía, vol. 39, núm. 153, abril-junio, 2008, pp. 159-183

Universidad Nacional Autónoma de México

Distrito Federal, México

Disponible en: http://www.redalyc.org/articulo.oa?id=11820161007

Cómo citar el artículo

Número completo

- Más información del artículo

Página de la revista en redalyc.org

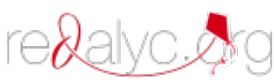

Sistema de Información Científica

Red de Revistas Científicas de América Latina, el Caribe, España y Portugal Proyecto académico sin fines de lucro, desarrollado bajo la iniciativa de acceso abierto 


\title{
CRISIS FINANCIERAS Y GLOBALIZACIÓN: UN ANÁLISIS DE SUS FACTORES DETERMINANTES
}

\author{
Arturo Rodríguez Castellanos* \\ Sara Urionabarrenetxea Zabalandikoetxea** \\ Nerea San Martín Albizuri***
}

Fecha de recepción: 6 de noviembre de 2007. Fecha de aceptación: 13 de marzo de 2008.

\section{Resumen}

El objetivo de este trabajo es identificar y analizar -mediante el planteamiento de un CD modelo conceptual explicativo- los factores que determinan la relación entre la globalización financiera y las crisis financieras. La principal característica diferenciadora de estos desequilibrios es su imprevisibilidad, al ser las expectativas autorrealizables y los efectos de contagio de las crisis entre distintos países las causas más importantes de la misma. Ahora bien, para el análisis de la posible vinculación entre las crisis y el proceso de globalización financiera es necesario estudiar otros dos aspectos: por una parte, la situación previa al inicio del proceso de integración en los mercados financieros globales, respecto del nivel de desarrollo económico y financiero de las diferentes naciones, y, por otra, la forma en que se desarrollan en ellos los procesos de apertura conducentes a dicha integración.

Palabras clave: globalización financiera, crisis financieras y efecto contagio.

* Director del Departamento de Economía Financiera II de la Universidad del País Vasco, España. Correo electrónico: arturo.rodriguez@ehu.es.

** Profesora en la Universidad del País Vasco, España. Correo electrónico: sara.urionabarrenetxea@ehu.es.

*** Becaria investigadora en la Universidad del País Vasco, España. Correo electrónico: nerea. sanmartin@ehu.es.

Agradecemos a dos evaluadores anónimos sus valiosas observaciones y sugerencias. Cualquier posible error debe ser atribuido exclusivamente a los autores. 


\section{Summary}

The objective of this study is to identify and analyze -by establishing an explicative conceptual model -the factors that determine the relationship between financial globalization and financial crises. The principal characteristic differentiating these imbalances is their unpredictability, because self-fulfilling expectations and the contagion effects between different countries are their most important causes. Meanwhile, in order to analyze the possible linkage between crises and the financial globalization process it is necessary to study two other aspects -on the one hand, the situation prior to the beginning of the process of integration in the global financial markets, in relation to the level of economic and financial development of the various countries, and, on the other hand, the ways in which opening processes conducive to this integration are developed within them.

Key words: financial globalization, financial crises, contagion effect, development, integration.

\section{Résumé}

L'objectif de ce travail est d'identifier et d'analyser - en posant un modèle conceptuel explicatif - les facteurs qui déterminent la relation entre la mondialisation financière et les crises financières. La principale caractéristique distinctive de ces déséquilibres est leur imprévisibilité, les expectatives étant auto-réalisables et l'effet de contagion des crises entre divers pays la cause principale de celles-ci. Or, pour l'analyse du lien possible entre la crise et le processus de mondialisation financière, il est nécessaire d'étudier deux autres aspects: d'une part, la situation préalable au début du processus d'intégration dans les marchés financiers mondiaux, c'est à dire le niveau de développement économique et financier des différentes nations, et d'autre part, comment se développent en leurs seins les processus d'ouverture qui mènent à l'intégration.

Mots clés: mondialisation financière, crises financières, effet de contagion, développement, intégration.

\section{Resumo}

O objetivo deste trabalho é identificar e analisar - através da proposta de um modelo conceptual explicativo - os fatores que determinam a relação entre a globalização financeira e as crises financeiras. A principal característica diferenciadora destes desequilíbrios é a sua imprevisibilidade, por serem as expectativas auto-realizáveis e os efeitos de contágio das crises entre distintos países as causas mais importantes da mesma. No entanto, para a análise da possível vinculação entre as crises e o processo de globalização financeira é necessário estudar outros dois aspectos: por um lado, a situação prévia ao início do processo de integração aos mercados financeiros globais, no que diz respeito ao nível de desenvolvimento econômico e financeiro das diferentes nações, e, por outro, a forma em que neles se desenvolvem os processos de abertura conduzentes a tal integração.

Palavras-chave: globalização financeira, crises financeiras, efeito contágio, desenvolvimento, integração. 


\section{Introducción}

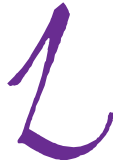

a globalización financiera es un fenómeno que se está desarrollando de forma progresiva desde la década de los ochenta del siglo pasado. Al coincidir aproximadamente con este horizonte temporal, las inestabilidades financieras han constituido un fenómeno que, de manera creciente, han azotado a numerosos países y regiones del mundo. Debido a las graves implicaciones que históricamente han tenido estas crisis para todo tipo de agentes — no sólo para las grandes empresas multinacionales, los inversionistas institucionales o las entidades financieras de gran tamaño, sino también para las empresas pequeñas y medianas e incluso para los inversionistas individuales-, se hace necesario un análisis exhaustivo de la posible vinculación entre las mismas y el proceso de globalización financiera.

Por ello, el objetivo propuesto en este trabajo es identificar y analizar los factores que determinan la relación entre la globalización financiera y las crisis financieras, mediante el planteamiento de un modelo conceptual explicativo de esos desequilibrios en la era de la globalización.

Las crisis financieras que han tenido lugar de forma paralela al desarrollo del proceso de globalización financiera parecen tener características que les diferencian de aquellas que se produjeron en épocas anteriores, siendo la imprevisibilidad la más destacable. Las causas más importantes de dicha imprevisibilidad son, por una parte, las expectativas autorrealizables (self-fulfilling expectations), y por otra los efectos de contagio de las crisis entre distintos países; ambos fenómenos, frecuentemente vinculados entre sí, parecen ser propios de la era de la globalización. Ahora bien, para el análisis de la posible vinculación entre las crisis y el proceso de globalización financiera es necesario analizar otros dos aspectos: por una lado, la situación, previa al inicio del proceso de integración en los mercados financieros globales, respecto del nivel de desarrollo económico y financiero de los diferentes países, y por otro la forma en que se desarrollan en ellos los procesos de apertura conducentes a dicha integración.

Con esta finalidad, en la segunda sección se analiza el concepto de globalización financiera. A continuación se destacan las características diferenciadoras de las crisis financieras en esta época de mercados financieros globalizados. Después se estudian los factores explicativos de las crisis financieras anteriormente indicadas, mediante el planteamiento de un modelo conceptual. Por último, se apuntan las conclusiones más relevantes y la bibliografía empleada. 
El proceso de globalización financiera:

algunas precisiones conceptuales

La globalización financiera se refiere al incremento de las relaciones globales por medio de los flujos financieros transnacionales (Prasad et al., 2003). Este proceso se está desarrollando por medio de un número creciente de vinculaciones de países o conjuntos de naciones a los mercados financieros internacionales, que recoge el concepto de integración financiera. El grado de integración depende de la medida en que se expone a las influencias externas (Beckers et al., 1996). Así, la integración de todas las naciones conduciría a la globalización, por lo que sería correcto utilizar indistintamente los términos de integración financiera internacional y globalización financiera; esta última es una integración financiera total, en su grado máximo, a escala mundial (Suzuki, 1990).

El acometimiento de los procesos de liberalización financiera que permitan la libre circulación internacional de los capitales financieros es un requisito imprescindible pero no suficiente para la precipitación del proceso de globalización financiera. Ésta exige, además del movimiento de flujos de capital transnacionales, la interconexión entre los mercados financieros de los países implicados, es decir, además de la movilidad internacional de activos, se requiere la sustitutividad, ${ }^{1}$ lo cual no se deriva de las liberalizaciones financieras.

Un proceso de integración financiera internacional exige, además de la pertinente liberalización, la concurrencia de al menos otros tres factores: en primer lugar, debido a que las liberalizaciones financieras no serían suficientes si no fuera posible un traslado rápido y sin excesivos costos adicionales, el avance en las tecnologías de la información y la comunicación ha contribuido decisivamente en el desarrollo del proceso de globalización financiera. En segundo, se ha avanzado, mediante la ingeniería financiera, en la creación y mejora de nuevos instrumentos financieros que permiten, por una parte, una mayor cobertura de los potenciales riesgos adicionales a los que el inversionista pueda exponerse en su actividad internacional y, por otra, un incremento de las operaciones de arbitraje y de especulación en el ámbito mundial, lo cual, en principio, debería tender a mejorar la eficiencia de los mercados financieros internacionales. Por último, el incremento, tanto en número como en patrimonio gestionado, de los inversionistas institucionales ha resultado de vital importancia

1 Los conceptos de movilidad y de sustitutividad internacional de activos fueron definidos por primera vez por Kenen (1976). 
puesto que cuentan con mejores recursos, tanto financieros como informacionales, para actuar en un entorno global. En caso de que los procesos de liberalización de distintos mercados financieros no fueran acompañados por los factores apuntados, éstos no conducirían a su integración.

Ahora bien, el rápido traslado internacional de los capitales que posibilitan los avances tecnológicos; la aparición de nuevos instrumentos financieros, que a menudo han contribuido en el incremento de las operaciones especulativas; y el aumento de inversionistas institucionales, cuyas operaciones han constituido un punto de referencia para muchos inversionistas no informados, a menudo han resultado fuente de inestabilidad (Eatwel y Taylor, 2005). Por tanto, la globalización financiera puede resultar más desestabilizadora que una liberalización financiera aislada.

\section{Las crisis financieras y sus características diferenciadoras en la era de la globalización}

Hay quien sostiene que desde el establecimiento del sistema de tipos de cambio flotantes en 1976 "la economía mundial está en crisis" (Berzosa, 1994). Quizá esta afirmación resulte excesiva para tomarla en sentido estricto, pero es ilustrativa para dejar patente la inestabilidad de la que es objeto la economía internacional desde que la liberalización de los flujos de capital se ha convertido en realidad. Según la Secretaría de Estado de Turismo y Comercio española, "es evidente que las crisis financieras no son nada nuevo, pero sí lo es su magnitud y su frecuencia. La mayor integración de los mercados reales y financieros —el fenómeno denominado globalización - ha aumentado de forma dramática la interrelación entre las economías y la capacidad de contagio de unas a otras" (Secretaría de Estado de Turismo y Comercio, 2004).

Las crisis acaecidas desde la década de los ochenta hasta la actualidad han sido especialmente frecuentes, y sus repercusiones se han hecho notar en zonas geográficamente muy lejanas al país en que se iniciaron dichos desequilibrios. Al objeto de observar si la globalización financiera trae consigo crisis financieras más frecuentes y de mayor intensidad, resulta interesante comparar periodos más globalizados financieramente con épocas de menor libertad de circulación internacional del capital. Bordo et al. (2001) realizan la comparación entre cuatro periodos distintos en función de esta dimensión: periodo de laissez-faire (1880-1914), considerado a menudo como la primera globalización financiera (Eichengreen y Bordo, 2002; Obstfeld y Taylor, 2002; Tugores, 2002; Martínez de Azagra y Wehbe, 2003), el periodo que transcurre entre las dos guerras mundiales, el periodo de Bretton Woods y, por último, el actual. 
De estos cuatro, el periodo anterior a 1914 y el correspondiente al marco de Bretton Woods resultan los más estables, teniendo sin embargo características muy diferentes. El primero corresponde a una época de libertad en la movilidad internacional del capital, mientras que en el sistema monetario internacional derivado de Bretton Woods la regulación de las relaciones internacionales era la nota predominante. Los más inestables son el periodo entre las dos guerras mundiales y el posterior a 1973. La inestabilidad vivida durante el periodo que abarca las dos guerras mundiales no es sorprendente, porque se trata de una época intrínsecamente inestable. Sin embargo, la inestabilidad de las últimas décadas merece especial atención, pues partiendo de una situación con características similares al primer periodo apuntado respecto de la libertad en la circulación internacional del capital, las crisis financieras sufridas son notablemente superiores.

Si se compara el último periodo — globalización actual— con el primero — primera época de globalización - se observa que las crisis presentes cuentan con una frecuencia de entre el doble y el triple que la del primer periodo. Por tanto, la extensión de la movilidad internacional del capital no parece ser suficiente para explicar la inestabilidad de los tipos de cambio y la alta frecuencia de las crisis financieras (Bordo et al., 2001). Ello sugiere, como se ha indicado anteriormente, que la globalización financiera viene acompañada por ciertos factores - sobre todo, el avance tecnológico, el aumento de los inversionistas institucionales y el desarrollo de la ingeniería financiera- que pueden contribuir a acentuar los efectos provocados por la liberalización financiera en dichas crisis.

Tradicionalmente, las economías se han comportado de manera diferente en vísperas de crisis; de esta forma, parecía viable prever las crisis financieras evaluando acontecimientos económicos anómalos que, en última instancia, constituirían las causas de los desequilibrios, o al menos un indicador temprano de su inminente desencadenamiento. Sin embargo, las crisis acaecidas en la era de la globalización financiera parecen ir acompañadas de un aumento de su imprevisibilidad respecto de épocas anteriores. ${ }^{2}$

$\mathrm{Al}$ atender el criterio que hace referencia a los procesos causales o desencadenantes de las distintas crisis financieras, la literatura referida al análisis de las mismas

2 Sin duda, todas las crisis tienen un cierto componente de imprevisibilidad. Pero las crisis en la era de la globalización financiera parecen manifestar con más intensidad esta característica, pues se producen en muchos casos en ausencia de un deterioro previo de los fundamentales económicos. En consecuencia, podría medirse a posteriori el grado de imprevisibilidad de las crisis comparando la evolución de los factores fundamentales de los países en las crisis anteriores y posteriores al desarrollo de la globalización financiera. 
puede dividirse en tres grandes bloques o grupos de modelos: los de primera generación, los de segunda generación y los de tercera generación.

Los denominados modelos de primera generación entienden las crisis como el resultado inevitable y previsible de la incompatibilidad entre las políticas económicas emprendidas por las autoridades de los países (en especial, políticas monetaria y fiscales expansivas junto a tipos de cambio fijos o casi fijos) y la debilidad de las variables económicas fundamentales en esas mismas naciones. ${ }^{3}$

Sin embargo, las crisis acaecidas en la era de la globalización financiera no parecen comportarse en acuerdo total con lo establecido por estos modelos, ${ }^{4}$ pues muchas de ellas se desencadenaron a pesar de que las variables económicas fundamentales no mostraban una situación que pudiera conducir a un inminente desequilibrio. Por ello, al objeto de explicar el surgimiento de las crisis actuales, surgen los modelos de segunda generación.

El primer modelo de segunda generación data de mediados de la década de los ochenta (Obstfeld, 1986) pero la mayoría de ellos surgieron tras la crisis europea (1992-1993) y la mexicana (1994-1995), vista la incapacidad de los modelos de primera generación para explicarlas. Según estos modelos, las crisis son fenómenos contingentes, no predecibles, y se producen por el cumplimiento de las expectativas de crisis generadas por los propios inversionistas. Obstfeld (1994) sostiene que "las anticipaciones especulativas dependen de las conjeturas sobre las respuestas gubernamentales, lo cual depende, a su vez, de las variaciones de los precios, que están en función de las expectativas de los inversores sobre la evolución futura de los tipos de cambio". Así, si se generalizan las expectativas sobre la depreciación de una moneda, los agentes económicos comienzan un ataque especulativo contra la misma, que las autoridades económicas del país emisor intentarán frenar mediante medidas con elevados costos para la actividad económica local: elevación de los tipos de interés, controles de capital, etcétera; ahora bien, como los agentes entienden que estas medidas no pueden extenderse indefinidamente (por los costos económicos antes mencionados), ello incrementa todavía más la especulación. Por tanto, se produce un círculo que, de no ser frenado con medidas adecuadas por las autoridades, puede derivar en crisis económica y consecuentes

3 Fueron Salant y Henderson (1978) quienes, por primera vez, hicieron uso de un modelo de primera generación, pero no fue hasta la aportación de Krugman en 1979 cuando tomó verdadera importancia. Posteriores autores han introducido mejoras en estos modelos: véase Flood y Garber (1984), Connolly y Taylor (1984), Calvo (1987), Edwards (1989), Krugman y Rotemberg (1991), Flood, Garber y Kramer (1996) y Dooley (1997).

4 Tal vez una excepción sea la crisis argentina de 2000-2001. 
inestabilidades financieras que no necesariamente deberían haber surgido, pero que aparecen porque los participantes del mercado así lo esperaban. ${ }^{5}$

Los modelos de tercera generación surgieron, a su vez, a partir de la incapacidad de los dos grupos de modelos anteriores para explicar satisfactoriamente el desencadenamiento de las crisis de los países asiáticos en 1997-1998, pues algunos de ellos presentaban desequilibrios y otros no; por otro lado, en estas naciones se produjeron con frecuencia "crisis gemelas" (twin crises), interactuando la crisis bancaria y la monetaria. Dentro de este grupo de modelos se explican dichos desequilibrios a partir de distintos factores, como el síndrome de sobreendeudamiento debido al desarrollo de situaciones de riesgo moral provocados, a su vez, por la aplicación de políticas gubernamentales inconsistentes (McKinnon y Pill, 1996; Dooley, 1997; Krugman, 1998; Corsetti et al., 1999); los problemas de agencia y de traslación de riesgos (Allen, 2001 y Requeijo, 2006); el pesimismo autorrealizable de los prestamistas internacionales (Chang y Velasco, 1998 y Radelet y Sachs, 1998) y el elevado endeudamiento empresarial en divisas (Krugman, 1999).

Por ello, puede comprobarse que los modelos tanto de segunda como de tercera generación suelen considerar, bien de forma exclusiva, bien como factores a tener en cuenta, fenómenos ajenos a variables fundamentales, y que en consecuencia aumentan el grado de imprevisibilidad de los desequilibrios. De hecho, la mayoría de las crisis desencadenadas en los últimos años no han sido previstas ni por los académicos expertos en crisis financieras, ni por los analistas financieros, ni por las agencias de rating y ni siquiera por el propio Fondo Monetario Internacional (FMI). Si bien los esfuerzos en la evaluación y seguimiento de la "salud" financiera internacional van en aumento, lo cierto es que en la década de los noventa se ha observado que la frecuencia y la extensión geográfica abarcada por las crisis son cada vez mayores, y, a pesar de los indicadores y diversos estudios para analizar sus causas a priori, la incapacidad para preverlas es manifiesta. ${ }^{6}$

5 Para posteriores modelos de segunda generación, véase Obstfeld (1996), Cole y Kehoe (1996, 2000), Sachs et al. (1996), Calvo (1998a, 1998b), Chang y Velasco (1998), Drazen (1998), y Jeanne (2000).

6 Aunque todavía es pronto para evaluar sus consecuencias, la reciente crisis bursátil y crediticia internacional desencadenada en agosto de 2007, derivada de la quiebra del mercado de las hipotecas subprime en EU, constituye otro ejemplo de imprevisibilidad: las principales agencias internacionales de rating mantuvieron hasta el último momento la máxima calificación crediticia a las emisiones de títulos respaldadas con este tipo de hipotecas, aunque el mercado inmobiliario en EU comenzó a presentar problemas ya en 2006. También es un ejemplo de "crisis sistémica", por su expansión global a partir de un país concreto, por medio de mercados financieros globalizados que han mostrado fallos de transparencia, y posiblemente del "comportamiento de rebaño", aspectos considerados más adelante. 
Al tener en cuenta estas características de las crisis, en la siguiente sección se propone un modelo comprensivo de la vinculación entre las crisis financieras y el proceso de globalización de los mercados financieros.

\section{Un modelo explicativo de las crisis}

\section{financieras en la era de la globalización}

\section{Esquema conceptual}

A mediados de la década de los ochenta Díaz-Alejandro (1985) auguró que la liberalización financiera traería consigo interminables inestabilidades financieras. Si bien, como ya se ha indicado en la sección anterior, la historia aporta evidencias suficientes como para eliminar la hipótesis de que las crisis financieras son fenómenos propios de la actual coyuntura liberalizadora, lo cierto es que desde el inicio del proceso de

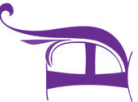
globalización de los mercados financieros, las crisis de origen fundamentalmente financiero han tenido una frecuencia sin precedentes y se han extendido con inusitada rapidez a mercados distintos de aquél en el que surgieron. Así, los desequilibrios acaecidos durante los últimos años "parecen mostrar una similitud principal, y es que parecen mantener cierta relación con la globalización de la economía mundial" (Bustelo et al., 1999).

Como se indicó en la segunda sección, el principal requisito que exige el fenómeno de la globalización financiera para su desarrollo es la previa liberalización de los mercados financieros. Los procesos de liberalización financiera han sido asociados con las crisis por numerosos autores (Demirgüç-Kunt y Detragiache, 1998; Banco Mundial, 1998; Kaminsky, Lizondo y Reinhart, 1998; Williamson y Mahar, 1998; Thompson, 1999; Aizenman, 2002). Ahora bien, las demás características vinculadas a la globalización financiera también pueden impulsar la creación o propagación de las crisis, siendo dos las principales: por una parte, los inversionistas institucionales, que gestionan un patrimonio cada vez mayor, pueden provocar graves inestabilidades cuando desplazan grandes volúmenes de capital de un país a otro, muchas veces fundamentados en razones financieramente no racionales; y, por otra, el desarrollo tecnológico, que posibilita el traslado de grandes volúmenes de capital de un país a otro sin demoras en el tiempo.

El propio FMI (1998) reconoce que existe cierta asociación entre liberalizaciones y crisis financieras. Esta institución, cuyas políticas van dirigidas precisamente al mantenimiento de la estabilidad económica y financiera, admite que los desequilibrios financieros de la década de los noventa, entre ellos el mexicano y el asiático, 
llegaron a tener efectos globales, añadiendo que éstos alcanzan una explicación en el contexto de los procesos de desregulación y liberalización financiera, el incremento de los flujos internacionales de capital y la innovación financiera de finales de siglo XX y principios del XXI. De hecho, todas las crisis acaecidas post-Bretton Woods fueron precedidas por importantes entradas de flujos exteriores de capital (Bustelo et al., 1999).

Ahora bien, la asociación entre liberalizaciones financieras y crisis financieras se ha producido fundamentalmente en los mercados emergentes. Con la excepción del caso europeo en 1992-1993, todos los desequilibrios en la década de los noventa del siglo pasado y en la década actual han afectado a economías emergentes. ${ }^{7}$ Este hecho es difícilmente atribuible al azar. Hutchison y Glick (2000) han observado que las crisis financieras más graves se han concentrado, sobre todo, en economías emergentes financieramente liberalizadas. Además, se ha observado que los primeros años de la apertura financiera suelen ser los más inestables (Kaminsky et al., 1998), cuando las economías nacionales aún no han desarrollado la capacidad de gestionar de manera adecuada tales aperturas y de asumir los riesgos adicionales que traen consigo.

Si las aperturas financieras perjudican principalmente a los países con mercados financieros emergentes, y las mayores probabilidades de crisis se dan durante los primeros años de apertura, ello parece estar vinculado con el nivel de desarrollo económico de tales países en el inicio de la apertura. Cuando el entorno institucional es sólido - adecuada regulación y supervisión de los intermediarios financieros, mercados financieros organizados, correcta aplicación de la legislación, bajo nivel de corrupción, ejecución contractual, etcétera- el impacto de la liberalización financiera sobre el sistema financiero es más débil (Demirgüç-Kunt y Detragiache, 1998). Por ello, antes de acometer las liberalizaciones resulta conveniente trabajar en la estabilización macroeconómica y la mejora de la supervisión institucional, dejando la liberalización financiera total para el final del proceso; esto es, durante el proceso de integración financiera una "represión financiera leve" tal vez resulte ventajosa (Williamson y Mahar, 1998).

Existen distintos factores que pueden terciar en la relación entre globalización financiera y crisis financieras.

7 Otra posible excepción puede ser la reciente derivada de las hipotecas subprime en EU, mencionada en la nota anterior. 
Figura 1

Modelo explicativo de las inestabilidades financieras basado en el proceso de globalización financiera

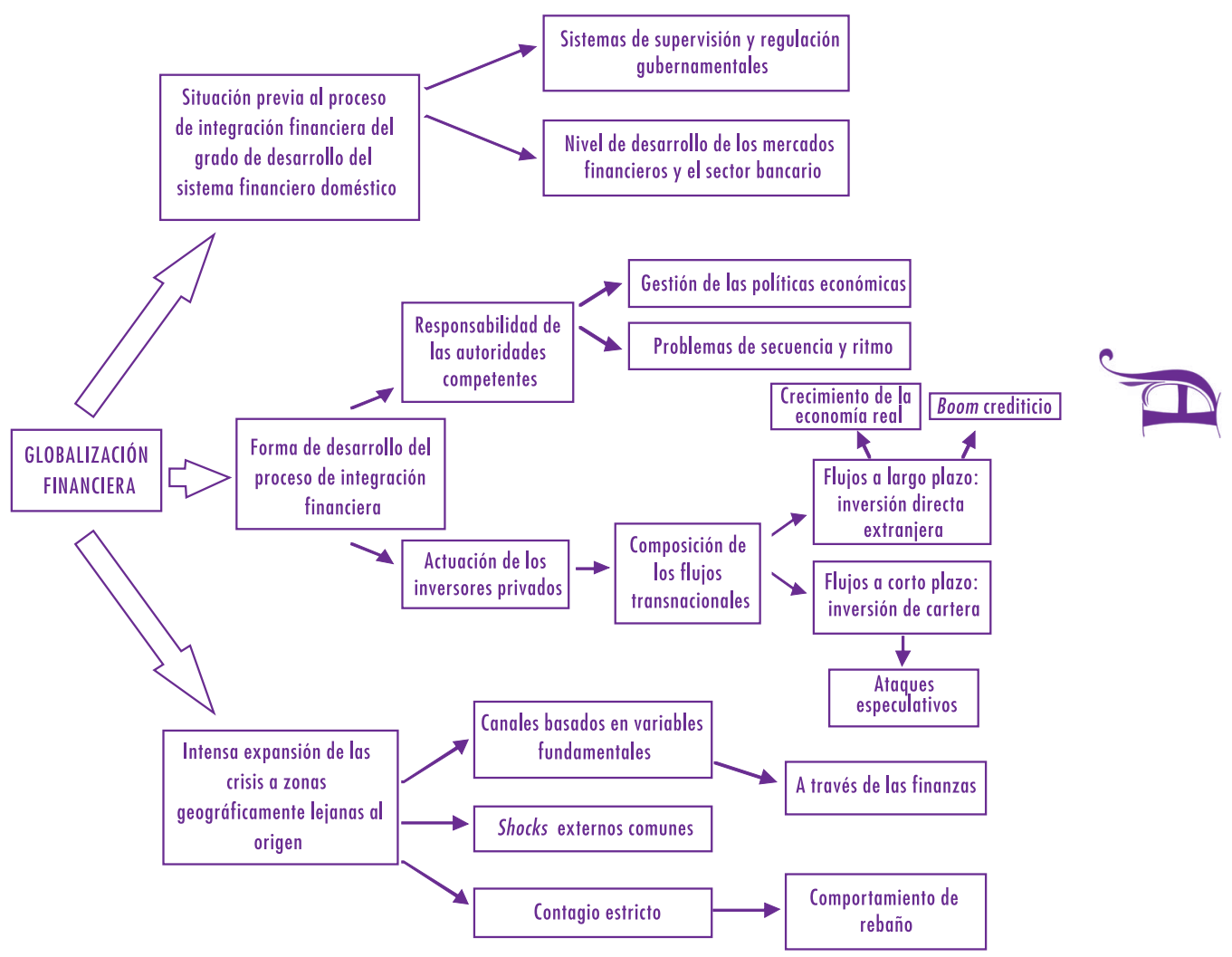

Fuente: Elaboración propia.

El proceso de integración de un país en los mercados financieros globales y — derivado de ello — su vulnerabilidad a las crisis puede desarrollarse de forma muy divergente, en función de las características que muestren cada uno de los siguientes aspectos:

- En primer lugar, la situación previa al inicio de la integración en los mercados financieros internacionales respecto del nivel de desarrollo económico y financiero del país o área geográfica a ser integrados en los mercados financieros internacionales. 
- En segundo, la forma en que se desarrolla el proceso de integración: tanto el sector público como el privado influyen en este aspecto.

- Por último, entre las distintas economías financieramente integradas pueden producirse efectos de contagio que pueden frenar u obstaculizar el desarrollo equilibrado del proceso de globalización.

No necesariamente —ni en todos los países, ni en la misma medida - estos tres aspectos deben manifestar características desfavorables. De hecho, en algunas crisis, como en las de los países asiáticos, ni la situación previa a la integración ni el proceso de la misma mostraban características desfavorables, pues la crisis se debía fundamentalmente, al parecer, al tercer aspecto, es decir, a los efectos de contagio, como se verá en la sección correspondiente a este factor. No obstante, la combinación simultánea de características negativas en los tres aspectos es la que provoca los efectos más devastadores.

Los aspectos apuntados, que serán analizados en los siguientes apartados, han afectado negativamente de forma especial a los países emergentes: en general, sus sistemas financieros estaban poco desarrollados en el momento de entrar en el proceso de integración; en muchos casos se han desarrollado políticas económicas equivocadas; los problemas de secuencia y ritmo han sido generalizados; los ataques especulativos por medio de los flujos a corto plazo han sido constantes, y los efectos de contagio se han propagado de forma espectacular.

\section{Situación previa al proceso de integración} en los mercados financieros globales

El grado de solidez del sistema financiero próximo a ser liberalizado condiciona de manera vital el éxito o el fracaso del proceso de apertura financiera. Así, para que el proceso transcurra correctamente es necesario contar con un sistema de supervisión y regulación gubernamentales adecuados y unos mercados financieros y un sector bancario suficientemente desarrollados (Chan-Lau y Chen, 2001).

Sistemas de supervisión y regulación financiera

En la inmensa mayoría de los países donde se han producido inestabilidades tras la apertura financiera - como ya se ha indicado, fundamentalmente países emergentes-, ésta se ha producido en un contexto de inadecuada regulación y supervisión prudencial del sector financiero, y en general de la actividad económica, por parte 
del gobierno (McKinnon y Pill, 1996; Dooley, 1997; Krugman, 1998; Corsetti et al., 1999; Singh, 1999). Así, a menudo las regulaciones financieras sobre requerimientos de capital no han resultado adecuadas para prevenir ni gestionar las inestabilidades financieras debido a su carácter procíclico, pues los requerimientos de capital impuestos a las entidades financieras aumentaban o se reducían según las variaciones del ciclo económico, ${ }^{8}$ de esta manera se explica la sobreconcesión de préstamos de los bancos y el sobreendeudamiento de las empresas en periodos de auge económico (Krugman, 1999). Además, los requerimientos de publicación de información empresarial y el sistema de clasificación de los activos bancarios por su nivel de calidad crediticia resultaban inadecuados. De hecho, autores como Mishkin (2001) consideran que una supervisión y una regulación endebles constituyen la principal causa de las inestabilidades financieras.

Desde su incorporación al proceso de globalización financiera, los países emergentes han experimentado en muchos casos una gran fragilidad financiera. En poco tiempo, estas naciones pasaron de tener una regulación estricta a una amplia liberalización de sus mercados, cuando todavía las infraestructuras financieras no eran adecuadas en el momento de liberalizar o no se adecuaron a tal efecto; es decir, las liberalizaciones se produjeron de forma tan rápida y masiva - a consecuencia, en muchas ocasiones, de fuertes presiones procedentes de los países avanzados y del propio FMI- que no se llevó a cabo un paralelo fortalecimiento de los mecanismos de supervisión y control; a ello también contribuyó cierto estado de opinión contrario al control y supervisión públicos, fruto de la extensión de la "filosofía de mercado", según la cual el mercado es el mejor controlador, pues proporciona toda la información necesaria para los sujetos operantes. En estas circunstancias, las entradas de capitales externos pueden debilitar aún más la salud del sistema financiero local.

Así pues, la mejora en la supervisión y regulación gubernamentales es deseable para que las entradas de capital no provoquen un boom crediticio y, como consecuencia, una asunción excesiva de riesgos de las instituciones bancarias.

8 El proceso es el siguiente: en la fase expansiva, los requerimientos de capital se reducen, lo cual provoca un incremento de la oferta de crédito y, consecuentemente, una acentuación de la expansión económica; por el contrario, en la fase recesiva, al aumentar el riesgo crediticio, los requerimientos de capital también aumentan, reduciendo el crédito disponible, pudiendo darse así el caso de que proyectos rentables no atraigan fondos para su financiación. La prociclicidad de los requerimientos de capital es un problema de difícil resolución, de hecho, ha sido uno de los aspectos de discusión sobre el Convenio de Basilea II (González, 2005). 
Nivel de desarrollo de los mercados

financieros y el sector bancario

Además de los sistemas de supervisión y regulación gubernamentales, un sistema financiero desarrollado requiere de unos mercados financieros y un sector bancario suficientemente desarrollados.

El nivel de desarrollo financiero se refiere al grado en que se realiza una asignación eficiente de los recursos financieros entre los distintos agentes de la economía. Cuanto más líquidos y transparentes sean los mercados financieros y cuanta mayor variedad de intermediarios financieros exista, mayor eficiencia se producirá en la asignación de los recursos. En relación con los mercados financieros, en las naciones emergentes es habitual que los estándares contables y los requerimientos de publicación sean bajos, con la consiguiente falta de transparencia y, en consecuencia, incertidumbre, principalmente, para inversionistas procedentes de países exteriores. En cuanto a los intermediarios financieros, como ya se ha indicado, en estas naciones es habitual que no cuenten con instrumentos adecuados para la evaluación y gestión de riesgos, o con responsables suficientemente formados en la valoración de riesgos y canalización de financiación. Ante estas debilidades, los flujos de capital procedentes de mercados internacionales, junto con la presión que supone la competencia en el ámbito internacional, pueden llevar a efectos desestabilizadores del sistema económico nacional.

\section{Modo de desarrollo del proceso}

de integración financiera del país

La forma en la que se desarrolla el proceso de integración financiera, tanto por parte de las autoridades competentes como de los inversionistas privados, condiciona los efectos positivos o negativos que se vayan a derivar de este proceso, así como la dimensión de estos efectos.

\section{Responsabilidad de las autoridades competentes}

La responsabilidad que asuman las autoridades competentes está referida, principalmente, a dos aspectos: por un lado, las políticas económicas acometidas para acompañar la liberalización financiera y, por otro, las cuestiones de secuencia y ritmo aplicadas al proceso.

Acerca de las políticas económicas aplicadas, éstas pueden constituir una de las causas tanto de éxito como de fracaso del proceso de integración financiera.

\section{DeSarrollo}


Así, respecto de las políticas monetarias, el control de la inflación y la influencia sobre el nivel de los tipos de interés y de cambio son los aspectos más estrechamente relacionados con la estabilidad financiera.

El hecho de alcanzar la estabilidad de los precios es una condición necesaria para tener una moneda sólida que facilite, por una parte, conseguir capital con deuda denominada en la moneda doméstica (Mishkin, 2001) y, por otra, la realización de inversiones locales.

En cuanto al segundo y tercer aspectos, algunos gobiernos, especialmente los de países emergentes, acometieron políticas referentes a los tipos de interés que, combinadas con regímenes de tipos de cambio fijos, indujeron a inestabilidades financieras: esos gobiernos tendieron a emitir deuda pública o a atraer capital extranjero mediante el establecimiento de altos tipos de interés, manteniendo, a su vez, la moneda doméstica ajustada al dólar o a una cesta de monedas (Kaminsky y Reinhart, 2000). Un primer efecto de esta situación fue que la entrada de los flujos de capital provocó una apreciación real de la moneda doméstica, lo cual hizo disminuir las exportaciones y, consecuentemente, erosionó el saldo de la cuenta corriente. Pero, además, en la mayoría de los casos se produjo un segundo efecto que hizo todavía más precaria la situación financiera: cuando se agotaron las reservas de divisas en un intento por suplir la disminución de entradas de fondos internacionales, y la política de altos tipos de interés no pudo mantenerse por una reducción en el ritmo de la economía, los inversionistas tendieron a desplazar masivamente sus fondos hacia otros países, con lo que la inestabilidad financiera resultó casi inevitable. ${ }^{9}$

Las políticas fiscales acometidas también pueden influir de modo significativo sobre el nivel de estabilidad en el desarrollo de un proceso de integración en los mercados financieros globales. De hecho, en las naciones donde ha habido más inestabilidades, tras el inicio de una liberalización financiera — como se ha indicado, sobre todo países emergentes-, los desequilibrios fiscales - desproporción entre gastos e ingresos públicos - han sido la nota predominante. Evi-

9 Con la experiencia de las crisis, y para evitar situaciones de este tipo, desde finales de la década de los noventa la acumulación de reservas en los países emergentes se ha multiplicado por tres, hasta acercarse a los cinco billones de dólares. En la actualidad, tales economías acumulan más de dos tercios de las reservas mundiales, y Asia es la principal impulsora de las políticas dirigidas en este sentido. Los superávit por cuenta corriente y las positivas entradas netas de flujos financieros explican en la mayoría de los casos tal acumulación (Rodrik, 2006; Alberola y Serena, 2007). 
dentemente, mantener a largo plazo tales desequilibrios puede debilitar el papel estabilizador del gobierno.

En cuanto a las cuestiones de secuencia y ritmo — denominados problemas de sustancia (Guitián y Varela, 2000) — en el desarrollo de las liberalizaciones financieras, existen dos cuestiones primordiales que habrán de considerarse: el cómo y el cuándo se llevan a cabo (Aizenman, 2002). De acuerdo con el momento temporal en el que se acomete el proceso y de la forma en que se desarrolla pueden derivarse consecuencias enormemente divergentes. En lo que se refiere a la secuencia, las autoridades con aspiración a liberalizar las economías de sus países deben plantearse cuestiones como la conveniencia de realizar primero la apertura de la cuenta corriente o la de capital, o la de eliminar primero las restricciones internas (sectores financieros nacionales) o las externas (los flujos financieros hacia el exterior). A pesar de que la literatura ha considerado que la reforma del comercio exterior es una precondición de la reforma financiera, en realidad se aprecia que no en todas las naciones se ha cumplido esta condición (Williamson y Mahar, 1998). El ritmo de apertura, por su parte, se refiere a la velocidad a la que se debe liberalizar el mercado de capitales.

Las respuestas a las cuestiones tanto de ritmo como de secuencia deben adecuarse a la situación concreta del país considerado; es decir, deben ser estudiadas en el seno de una economía concreta, pues ambas dependen de la situación y características de la economía, sin ser aceptables las soluciones generalizadas.

A los problemas de sustancia se les puede añadir el problema de procedimientos, consistente en la necesidad de establecer ciertos acuerdos entre todos los países sobre un régimen internacional de normas directrices para los movimientos de capital, que sea lo suficientemente flexible como para controlar todas las situaciones concretas que se puedan presentar.

Actuación de los inversionistas privados

Tras un proceso de liberalización financiera, el país recibe distintos tipos de flujos financieros. El criterio más importante de su clasificación, con el fin de evaluar su posible efecto en las inestabilidades financieras, es su horizonte temporal: pueden tener un vencimiento a largo o a corto plazos.

Los flujos de capital a largo plazo suelen ser bastante estables y por lo regular no producen repentinas retiradas masivas, muchas veces basadas en un comportamiento de manada, como sucede con los flujos a corto plazo. En general, se les ha reconocido su capacidad para ejercer una influencia positiva en el desarrollo econó- 
mico a largo plazo, puesto que este tipo de flujos, al estar dirigidos preferentemente hacia la inversión real, pueden implicar transferencias tecnológicas, mejora de la calidad de los inputs y mejoras en la formación del capital humano. Según Boyer (1999), si la afluencia de estos capitales externos es constante al menos durante una década y los indicadores macroeconómicos estables, las exportaciones y el crecimiento económico aumentarán; pero si los efectos negativos, como la alta volatilidad o inestabilidad, se han producido antes de la modernización tecnológica e industrial, la liberalización financiera provocará intensas crisis con una gran capacidad de expansión. ${ }^{10}$

A pesar de la potencial capacidad de los flujos a largo plazo para inducir un crecimiento económico, su excesivo volumen puede conducir a graves inestabilidades. Como ya se ha indicado, resulta habitual que tras un proceso de liberalización financiera se produzca una sobreextensión del crédito bancario (lending boom), lo cual reduce la calidad de los activos bancarios y crea una falta de rigor en la asunción de riesgos de los intermediarios financieros a la hora de conceder préstamos.

Este riesgo se puede trasladar a las empresas si la disponibilidad de excesivo crédito contribuye a la sobreinversión privada, lo cual deriva en una reducción del rendimiento del capital y la eficiencia de la inversión (Bustelo et al., 1999).

Tal como sostiene Mishkin (2005), la globalización financiera no es inherentemente buena ni mala, sino que se le pueden asociar efectos positivos basados en el crecimiento económico o negativos vinculados con inestabilidades financieras. Así pues, se deben sopesar estos dos efectos contradictorios de la liberalización de los capitales a largo plazo. La cuestión reside en cuál de ellos supera al otro. Los escasos estudios empíricos realizados sobre el tema no muestran consenso: mientras que autores como Demirgüç-Kunt y Detragiache (1998) observan que el posible crecimiento económico provocado por los procesos de liberalización es anulado por la existencia de crisis financieras, y, por tanto, los efectos positivo y negativo parecen mostrar aproximadamente la misma importancia; otros como Tornell et al. (2004), sostienen que los beneficios obtenidos a partir del crecimiento económico superan los perjuicios ocasionados por las crisis.

10 Ahora bien, también hay quienes, como Tobin (2000), restan toda importancia al posible efecto favorecedor que puede provocar la afluencia externa de capitales, aunque sean a largo plazo, puesto que defienden que la mayoría de las inversiones que han impulsado el crecimiento de los países procede del ahorro interno y no del externo. 
Aunque los flujos de capital transnacionales a largo plazo puedan crear inestabilidad financiera, sin duda los de corto plazo tienen mucha mayor capacidad para ello. Los flujos de capital que se dirigen a mercados financieros exteriores para diversificar las carteras de acciones y obligaciones, o con fines especulativos, son los que provocan la mayor parte de las inestabilidades.

La mayoría de las entradas de capital a las economías emergentes son a corto plazo, es decir, son inversiones mayoritariamente especulativas que provocan efectos desestabilizadores y, en última instancia, crisis financieras (Boyer, 1999; Tobin, 2000). De hecho, una de las principales causas de las crisis de Asia, Latinoamérica y Rusia de la década de los noventa fue la libertad de circulación de los flujos de capital a corto plazo, debido a su alta volatilidad y a las repentinas retiradas masivas que se produjeron tras el inicio de las crisis (Singh, 2002).

El propio Köhler (2000), exdirector gerente del FMI, reconoce que la volatilidad de los flujos financieros externos hacia los mercados emergentes en los últimos años ha sido intensa y que se espera que continúe siéndolo en el futuro inmediato. Teniendo en cuenta que esta volatilidad es "fuente de turbulencia y crisis, $[\ldots]$ los mercados emergentes deben mantener el impulso de la reforma estructural y procurar que su situación macroeconómica sea lo más sólida posible”.

\section{Alta expansión de las crisis financieras}

en las fronteras geográficas

Las crisis financieras acaecidas en la era de la globalización cuentan con un factor común: muestran una gran capacidad de expansión a lo largo de las fronteras geográficas (Kaminsky y Reinhart, 2003). La globalización propicia esta expansión debido, sobre todo, a los sunspots o contagios no relacionados con variables fundamentales. Así, el contagio propiamente dicho, o "contagio estricto", se refiere al comovimiento sufrido por los países durante los periodos de crisis que no puede ser explicado por las variables económicas fundamentales (Gregorio y Valdés, 2001; Bekaert et al., 2003; Wongswan, 2003).

Un principio básico de la teoría económica clásica es que las decisiones de inversión reflejan las expectativas de los inversionistas formadas racionalmente, y por tanto las decisiones son adoptadas en función de toda la información disponible de una manera eficiente. Sin embargo, en la realidad práctica no siempre sucede así, sino que las decisiones de inversión pueden ser conducidas por la psicología de grupo. De hecho, una parte muy importante de los contagios producidos durante las crisis en la era de la globalización financiera no son atribuibles a cambios en los 
factores fundamentales ni tampoco a un shock externo común, ${ }^{11}$ sino simplemente a la "psicología", "actitud" o "comportamiento" del inversionista.

Una de las manifestaciones más claras de este fenómeno es el "comportamiento de rebaño" (herd behavior). ${ }^{12}$ Como sostienen Calvo y Mendoza (2000), este comportamiento puede ser racional hasta cierto punto, en el sentido de que la globalización de los mercados financieros reduce el incentivo de los inversionistas para buscar información de primera mano y los anima a seguir estrategias de inversión comunes.

Tal como indicaba Keynes (1936:316), "los especuladores están más preocupados en prever el próximo cambio en el sentimiento del mercado que en calcular una estimación razonable del rendimiento futuro de los activos", lo cual ha hecho que la especulación en el ámbito internacional se haya convertido en una práctica habitual.

A ello hay que añadir la forma en la que se lleva a cabo esta especulación: gracias a la globalización, el número de mercados en los que se invierte aumenta y, por tanto, disminuye la proporción de los activos de un país en la cartera de un inversionista. Así, la posibilidad real de obtener información cercana y completa — como se pueda hacer en un mercado doméstico - disminuye notablemente y, además, la ventaja de recopilar información sobre un país específico se reduce, debido a la disminución de su proporción en la cartera compuesta. Por tanto, es habitual que se intensifique el "comportamiento de rebaño" entre los distintos inversionistas, como sucede, con frecuencia, cuando las expectativas se forman en un contexto en que la información es asimétrica e imperfecta (Calvo y Mendoza, 2000).

Si se observan ciertos movimientos de algunos inversionistas supuestamente informados —en general, inversionistas institucionales_- gran parte de los no informados actuarán de la misma manera, no basando su decisión en razonamientos según criterios racionales. Las reacciones son tanto más acusadas cuanto más a corto plazo

11 La expansión de las crisis financieras a lo largo de las fronteras geográficas puede basarse en los spillovers - efectos relacionados con factores fundamentales- (De la Dehesa, 2000b; Fratzscher, 2002; Caramazza et al., 2004) o en los monsoonal effects —shocks externos comunes-(Caramazza et al., 2004), además del contagio estricto, no relacionado con variables fundamentales — sunspots —; ahora bien, es este último el que caracteriza la era de la globalización financiera.

12 La hipótesis del "comportamiento de rebaño", ya reflejado por Keynes y que ha sido tan importante en el contagio de las crisis financieras, ha sido desarrollada formalmente por Gwynne (1986), Scharfstein y Stein (1990) y Banerjee (1992). 
es el vencimiento de los flujos de capital. Este tipo de comportamiento es el que produce el efecto bandwagon: se da una exagerada desproporción entre la entidad de los factores desencadenantes y su alcance. ${ }^{13}$

Goldstein (1998) atribuye el hecho de que la mayoría de las crisis financieras se desarrollen en forma de clusters no a un efecto contagio propiamente, sino al efecto wake up call o "de aviso". Según este autor, algunas crisis son consideradas por los inversionistas como un aviso de que en economías con características similares pueda suceder lo mismo y, por tanto, retiran de ellas sus capitales de manera preventiva.

No puede despreciarse, en la propagación de los contagios, la contribución de los nuevos instrumentos financieros producto de la ingeniería financiera, que a menudo ha incrementado las operaciones especulativas, y el aumento de inversionistas institucionales (en especial hedge funds), cuyas operaciones, al constituir referencia para los inversionistas no informados, han resultado fuente de inestabilidad (Eatwel y Taylor, 2005).

Es posible que la distinción entre el efecto "contagio puro" y el "de aviso" sea puramente terminológica. La realidad es que en las crisis recientes el contagio se ha producido; así, países que en principio no mostraban señales de debilidad macroeconómica alguna se han visto altamente involucrados, como ocurrió en la crisis asiática. Este desequilibrio estalló en Tailandia en julio de 1997 cuando se produjo una devaluación del bath tailandés. Hasta ese momento el crecimiento económico había sido rápido y sostenible, y las políticas desarrolladas habían sido consideradas como ejemplo del buen hacer; así pues, tanto inversionistas como analistas financieros fueron sorprendidos por esta crisis. Ahora bien, hay que tener en cuenta que en aquel momento el país contaba con un sistema financiero poco sólido, alto déficit por cuenta corriente y sobrevaloración de los activos bursátiles. Los efectos de la crisis se propagaron con rapidez a otras naciones de la región, sobre todo Filipinas, Indonesia y Malasia, y poco tiempo después a Hong Kong, Corea del Sur, e incluso a Japón y a Rusia, que ni siquiera contaban con las mismas debilidades de los países en los que se inició la crisis, pero igualmente sufrieron los efectos del contagio.

13 En los mercados emergentes, donde la información es menos transparente y más asimétrica, se producen en mayor grado errores de juicio y actuaciones irracionales en forma de percepciones o intuiciones. Así, los sentimientos de euforia y depresión o pánico se suelen producir más frecuentemente, por lo que el contagio tiene mayores probabilidades de que ocurra en dichos mercados. 
Es claro, pues, que el contagio por medio de los flujos financieros internacionales se ha convertido en uno de los aspectos clave de los riesgos originados por la liberalización y globalización financieras (De la Dehesa, 2000a, 2000b).

Muchos autores asimilan las crisis de tercera generación a crisis de tipo sistémico (Palma, 1998; Cartapanis, 2004). En efecto, si son desequilibrios donde el efecto contagio inducido por las entidades y los mercados financieros juega un papel fundamental, éstos son también los componentes básicos del riesgo sistémico. Así, es frecuente asignar este tipo de crisis, en especial las de los países asiáticos, al paradigma de riesgo sistémico: fallos de los mercados financieros (riesgo moral, selección adversa, falta de coordinación), ataques especulativos autorrealizables seguidos de quiebras bancarias en cadena, hundimiento de la actividad económica, etcétera. El incremento del riego sistémico es una característica resaltable de la globalización financiera, y, como la reciente crisis financiera internacional ha mostrado, no es exclusiva de las economías emergentes.

\section{Conclusiones}

Las conclusiones que se desprenden del trabajo realizado son las siguientes:

- En la era de la globalización financiera, y respecto a épocas anteriores, la imprevisibilidad de las crisis se ha incrementado. Así, en esta era la previsión de las crisis mediante el análisis de las variables económicas fundamentales plantea mayores dificultades; más bien, las crisis parecen asociarse a las expectativas de los inversionistas basadas en criterios de psicología de grupo que, a menudo, se alejan de la racionalidad financiera.

- El grado de solidez del sistema financiero próximo a ser liberalizado condiciona de forma decisiva el éxito o el fracaso del proceso de apertura financiera. Si los mercados financieros y los organismos de supervisión y regulación no se encuentran suficientemente desarrollados, tendrán menos capacidad para responder a posibles ataques especulativos provenientes de inversionistas exteriores. Por ello, es conveniente que los sistemas financieros y la economía en general cuenten con un nivel de desarrollo mínimo antes de abrirse a los mercados internacionales.

- La forma en que se desarrolla el proceso de integración financiera condiciona los efectos positivos o negativos que se vayan a derivar de éste, así como la dimensión de estos efectos. Tanto el sector público como el privado influyen en este aspecto: por una parte, las autoridades competentes tienen la responsabilidad de orientar el proceso mediante el establecimiento de políticas económicas adecuadas y también 
el ritmo y la secuencia de apertura de los mercados. Si éstos no resultan acertados, en lugar de impulsar el buen desarrollo de la apertura constituirán escollos a superar. Por otra, las características de los flujos recibidos en el país procedentes de inversionistas internacionales condicionan su efecto positivo o negativo: mientras que los flujos a largo plazo pueden constituir una buena oportunidad de crecimiento de la economía real, los de vencimiento a corto pueden ser fuente de grandes inestabilidades.

- Entre las distintas economías financieramente integradas se producen efectos de contagio de situaciones de inestabilidad, originados en factores no relacionados con la evolución de las variables económicas fundamentales, sino más bien con las características de las entidades y los mercados financieros y su conexión con los mercados financieros globales, generando un riesgo sistémico que resulta un elemento fundamental en el desarrollo de muchas de las crisis habidas en esta era.

- Las consecuencias de lo anterior para los agentes privados que se hallan involucrados en procesos de internacionalización son:

- Respecto de la anticipación de las crisis, dada su imprevisibilidad, antes que modelos de predicción deben emplearse instrumentos para el análisis y la gestión de los riesgos inherentes a cada entorno, así como mecanismos para reaccionar adecuadamente ante la aparición de inestabilidades, teniendo en cuenta su posible contagio entre países.

- En la consideración de la inversión en un determinado país, resulta fundamental el análisis previo del grado de solidez de su sistema financiero y de la adecuación de los organismos de supervisión y regulación del mismo, así como el grado en que se halla el proceso de su integración en el sistema financiero global, y la adecuación de las políticas y regulaciones para la realización de dicho proceso.

- No obstante, la reciente crisis de las hipotecas subprime estadounidenses, que pronto se ha convertido en crisis internacional, muestra cómo, incluso en países con mecanismos de supervisión financiera aparentemente efectivos, pueden originarse circunstancias de sobrecrédito y baja transparencia que, en mercados financieros globalizados, conduzcan a una crisis sistémica. 


\section{Bibliografía}

Aizenman, J., "Financial opening: evidence and policy options", NBER Working Paper, núm. 8900, Cambridge, 2002, pp. 27.

Alberola, E. y J. M. Serena, "Global financial integration, monetary policy and reserve accumulation. Assessing the limits in Emerging Economics", Moneda y Crédito, núm. 224, 2007, pp. 105-152.

Allen, F., "Financial structure and financial crisis", International Review of Finance, vol. 2: 1/2, 2001, pp. 1-19.

Banco Mundial, Global economic prospects, Washington, diciembre de 1998.

Banerjee, A., "A simple model of herd behaviour", Quarterly Journal of Economics, vol. 107, núm. 3, agosto de 1992, pp. 797-817.

Barberá, R.A. y M. Blanca, "La interpretación de las crisis financieras a través de la literatura académica”, Boletín Económico de ICE, núm. 816, 2004, pp. 9-21.

Beckers, S., G. Connor y R. Curds, "National versus global influences on equity returns", Financial Analysts Journal, vol. 52, núm. 2, marzo-abril de 1996, pp. 31-39.

Bekaert, G. y C. R. Harvey, "Market integration and contagion", NBER Working Paper, núm. 9510, Cambridge, 2003, pp. 33.

Berzosa, C., La economía mundial en los 90, tendencias y desafíos, Barcelona, Icaria, 1994.

Bordo, M., B. Eichengreen; D. Klingebiel y M. S. Martínez-Peira, "Is the crisis problem growing more severe?", Economic Policy. A European Forum, vol. 16, núm. 32, abril de 2001, pp. 53-82.

Boyer, R., "Dos desafíos para el siglo XXI: disciplinar las finanzas y organizar la internacionalización", Revista de la CEPAL, núm. 69, diciembre de 1999, pp. 33- 51.

Bustelo, P., C. García y L. Olivié, "Global and domestic factors of financial crises in emerging economies: lessons from the East Asian episodes (1997-1999)", ICEI Working Paper, núm. 16, 1999, pp. 106.

Calvo, G. A., "Balance of payments crises in a cash-in-advance economy", Journal of Money, Credit, and Banking, vol. 19, núm. 1, 1987, pp. 19-32.

,"Balance of payments crises in emerging markets. Large capital inflows and sovereign governments", Working Paper, University of Maryland, 1998a.
"Capital flows and capital-market crises: the simple economics of sudden stops", Journal of Applied Economics, vol. 1, núm.1, 1998b, pp. 35-54.

y E. Mendoza, "Rational contagion and the globalization of securities markets", Journal of International Economics, vol. 51, núm.1, 2000, pp. 79-113.

Caramazza, F., L. Ricci y R. Salgado, "International financial contagion in currency crises", Journal of International Money and Finance, vol. 23, núm.1, 2004, pp. 51-70.

Cartapanis, A., "Le déclenchement des crises de change: qu'avons-nous appris depuis dix ans?", Économie Internationale, vol. 97, 2004, pp. 5-48.

Chang, R. y A. Velasco, "Financial crises in emerging markets: a canonical model", Federal Reserve Bank of Atlanta Working Paper, núm. 98-10, julio de 1998, pp. 51.

Chan-Lau, J. A. y Z. Chen, "Crash-free sequencing strategies for financial development and liberalization", IMF Staff Papers, vol. 48, núm. 1, 2001, pp. 179-196.

Cole, H. L. y T. J. Kehoe, "A self-fulfilling model of Mexico's 1994-1995 debt crisis", Journal of International Economics, vol. 41, núm. 3-4, 1996, pp. 309-330.

"Self-fulfilling debt crises", Review of Economic Studies, vol. 67, núm. 1, enero de 2000, pp. 91-116.

Connolly, M. B. y D. Taylor, "The exact timing of the collapse of an exchange rate regime and its impact on the relative price of trades goods", Journal of Money, Credit and Banking, vol. 16, núm. 2, 1984, pp. 194-207.

Corsetti, G., P. Pesenti y N. Roubini, "What caused the Asian currency and financial crisis?", Japan and the World Economy, vol. 11, núm. 3, 1999, pp. 305-373.

De la Dehesa, G., Comprender la globalización, Madrid, Alianza, 2000a.

"El papel de los mercados financieros en la autoalimentación y contagio de las crisis financieras", Moneda y Crédito, núm. 210, 2000b, pp. 11-58.

Demirgüç-Kunt, A. y E. Detragiache, "Financial liberalization and financial fragility", IMF Working Paper, núm. 83, 1998, pp. 53. 
Díaz-Alejandro, C., "Good-bye financial repression, hello financial crash", Journal of Development Economics, vol, 19, núm. $1-2,1985$, pp. 1-24.

Dooley, M. P., "A model of crises in emerging markets", NBER Working Paper, núm. 6300, Cambridge, 1997, pp. 35.

Drazen, A., Political contagion in currency crisis, University of Maryland, Mimeo, 1998.

Eatwell, J. y L. Taylor, Finanzas globales en riesgo: un análisis a favor de la regulación internacional. Buenos Aires, Siglo XXI Editores, 2005.

Edwards, S., Real exchange rates, devaluation and adjustment: Exchange rate policy in developing countries, Cambridge, MIT Press, 1989.

Eichengreen, B. y M. D. Bordo, "Crises now and then: what lessons from the last era of financial globalization?", NBER Working Paper, núm. 8716, Cambridge, 2002, pp. 55.

Flood, R. P. y P. M. Garber, "Collapsing exchange rate regimes: some linear examples" Journal of International Economics, vol. 29, núm. 1, 1984, pp. 1-13.

y C. Kramer, "Collapsing exchange rate regimes: Another linear example", Journal of International Economics, vol. 41, núm. 3-4, 1996, pp. 223-234.

Fondo Monetario Internacional, "Los movimientos de capital en el marco de una enmienda del Convenio Constitutivo del FMI", Informe Anual del Directorio Ejecutivo Correspondiente al Ejercicio Cerrado el 30 de Abril de 1998, cap. 10, Washington.

Fratzscher, M., "On currency crises and contagion", International Journal of Finance and Economics, vol. 8, núm. 2, 2003, pp 109-129.

Goldstein, M., The Asian financial crisis: causes, cures, and systematic implications, Washington, Institute for International Economics, 1998.

González, E., "Prociclicidad, volatilidad financiera y Basilea II", Revista de Estabilidad Financiera, Banco de España, núm. 8, pp. 153-161.

Gregorio, J. y R. O. Valdés, "Crisis transmission: evidence from the debt, Tequila, and asian Flu crises", en K. Forbes y S. Claessens (eds.), International financial contagion, Massachusetts, Kluwer Academic Publishers, 2001, pp. 99-128.

Guitián, M. y F. Varela, Sistemas financieros ante la globalización, Madrid, Pirámide, 2000.
Gwynne, S., Selling money, Nueva York, Weindenfeld \& Nicholson, 1986.

Hutchison, M. M. y R. Glick, "Banking and Currency Crises: How Common are Twins?", UCSC Dept. of Economics Working Paper, núm. 488, enero de 2000, pp. 43.

Jeanne, O., "Currency crises: A perspective on recent theoretical developments", Special Papers in International Economics, núm. 20, Princeton University, International Finance Section, 2000.

Kaminsky, G. L. y C. M. Reinhart, "Crisis financieras en Asia y Latinoamérica: ahora y entonces", en M. Guitián y F. Varela (eds.): Sistemas financieros ante la globalización, Madrid, Pirámide, 2000, pp. 149-151.

"The center and the periphery: the globalization of financial turmoil", NBER Working Paper, núm. 9479, Cambridge, 2003, pp. 47.

y S. Lizondo, "Leading indicators of currency crises", IMF Staff Papers, núm. 45, 1998, pp. 48.

Kaufman, G., "Banking and currency crises and systemic risk: A taxonomy and review", Financial Markets, Institutions and Instruments, vol. 9, núm 2, 2000a, pp. 69-131.

"Banking and currency crises and systemic risk: Lessons from recent events", Federal Reserve Bank of Chicago Economic Perspectives, vol. 24, núm 3, 2000b, pp. 9-28.

Kenen, P., Capital mobility and financial integration: a survey, Nueva Jersey, Princeton University Press, 1976.

Keynes, J. M., The general theory of employment, interest and money, Nueva York, Hartcourt, Brace and Co., 1936.

Köhler, H., "En París, Köhler insta a mantenerse alerta para prevenir futuras crisis financieras", FMI Boletín, 12 de junio de 2000, pp. 180-182.

Krugman, P., "A model of balance-of-payments crises", Journal of Money, Credit and Banking, vol. 11, núm. 3, 1979, pp. 311-325.

, "What happened to Asia?", 1998, www.princeton.edu/ pkrugman, pp. 1-11, consultado el 27 de febrero de 2006.

, "Balance sheets, the transfer problem, and financial crises", 1999, www.mit.edu/ krugman, pp. 1-23, consultado el 28 de febrero de 2006.

y J. Rotemberg, "Speculative attacks on target zones", en P. Krugman y M. Miller (eds.): Target Zones and Currency Bands, Oxford, Oxford University Press, 1991.

Vol. 39, núm. 153, abril-junio / 2008 
Marshall, D., "Understanding the Asian crisis: Systemic risk as coordination failure", Federal Reserve Bank of Chicago Economic Perspectives, vol. 22, núm 3, 1998, pp. 13-28.

Martínez de Azagra, L. y C. D. Wehbe, "La organización económica internacional y los problemas derivados de la globalización”, en F. González y J. G. Sequeiros (eds.), Orden económico mundial: globalización y desarrollo, Coruña, Netbiblo, 2003, pp. 229-244.

Mckinnon, R. y H. Pill, "Credible liberalization and international capital flows. The overborrowing syndrome", en T. Ito y A. Krueger (eds.), Financial Regulation and Integration in East Asia, Chicago, Chicago University Press, 1996, pp. 7-42.

Mishkin, F. S., "Financial policies and the prevention of financial crises in emerging market countries", NBER Working Paper, núm. 8087, Cambridge, 2001, pp. 40.

"Is financial globalization beneficial?", NBER Working Paper, núm. 11891, Cambridge, 2005, pp. 52.

Obstfeld, M., "Rational and self-fulfilling balance-of-payments crises", American Economic Review, vol. 76, núm. 1, 1986, pp. 72-81.

"The logic of currency crises", $\mathrm{Ca}$ hiers Économiques et Monétaires, núm. 43, 1994, pp. 189-213.

, "Models of currency crises with selffulfilling features", European Economic Review, vol. 40, 1996, pp. 1.037-1.047.

y A.M. Taylor, "Globalization and capital markets", NBER Working Paper, núm. 8846, Cambridge, 2002, pp. 67.

Palma, G., "Three and a half cycles of "mania, panic, and [asymmetric] crash': East Asia and Latin America compared", Cambridge Journal of Economics, vol. 22, núm. 6, noviembre de 1998; pp. 789-808.

Prasad, E.; K. Rogoff; S. Wei y M. A. Kose, "Effects of financial globalization on developing countries: some empirical evidence", IMF Occasional Paper, núm. 220, septiembre de 2003, pp. 86.

Requeijo, J., Anatomía de las crisis financieras, Madrid, MacGraw-Hill, 2006.

Rodrik, D., "The social cost of foreign exchange reserves". International Economic Journal, vol. 20, núm. 3, 2006, pp. 253-266.

Sachs, J.; A. Tornell y A. Velasco, "Financial Crises in emerging markets: The lessons from 1995", Brookings Papers on Economic Activity, vol. 1, 1996, pp. 147-215.
Salant, S. y D. Henderson, "Market anticipation of government policy and the price of gold", Journal of Political Economy, vol. 86, núm. 4, 1978, pp. 627-648.

Scharfstein, D. y J. C. Stein, "Herd behaviour and investment", American Economic Review, vol. 80, núm. 3, 1990, pp. 465-479.

Secretaría de Estado de Turismo y Comercio (Ministerio de Industria, Turismo y Comercio), "El FMI y la Estabilidad Financiera", Área de Comercio Exterior, 10 de diciembre de 2004, http://www.mcx.es.

Shirakawa, M; K. Okina y S. Shiratsuka, "Financial market globalization: present and future", Institute for Monetary and Economic Studies Discussion Paper Series, núm. 97-E-11, 1997, pp. 1-60.

Singh, A., "Asian capitalism and the financial crises", en J. Michie y J. G. Smith (eds.): Global instability: the political economy of world economic governance, Londres, Routledge, 1999, pp. 9-36.

, "Capital account liberalization, free long-term capital flows, financial crises and economic development", ESRC Centre for Business Research Working Paper, núm. 245, University of Cambridge, 2002, pp. 32.

Suzuki, Y., "Increasing the financial integration of US and Japanese markets", Japan and the World Economy, vol. 2, núm. 4, 1990, pp. 387-395.

Thompson, G., "Globalización", Revista Internacional de Ciencias Sociales, núm. 160, junio de 2000, www.unesco.org, consultado el 22 de enero de 2007.

Tobin, J., "Financial globalization", World Development, vol. 28, núm. 6, 2000, pp. 1.101-1.104.

Tornell, A.; F. Westermann y L. Martínez, "The positive link between financial liberalization growth and crises", NBER Working Paper, núm. 10293, Cambridge, 2004, pp. 53.

Tugores, J., Economía internacional, globalización e integración regional, Madrid, McGraw-Hill, 2002.

Williamson, J. y M. Mahar, A Survey of Financial Liberalization, Nueva Jersey, Princeton University, 1998.

Wongswan, J., "Contagion: an empirical test", FRB International Finance Discussion Paper, núm. 775, 2003, pp. 39. 